\title{
TRANSFORMATIONS ON WHITE NOISE FUNCTIONS ASSOCIATED WITH SECOND ORDER DIFFERENTIAL OPERATORS OF DIAGONAL TYPE
}

\author{
DONG MYUNG CHUNG ${ }^{1}$, UN CIG JI AND NOBUAKI OBATA ${ }^{2}$
}

\begin{abstract}
A generalized number operator and a generalized Gross Laplacian are introduced on the basis of white noise distribution theory. The equicontinuity is examined and associated one-parameter transformation groups are constructed. An infinite dimensional analogue of $a x+b$ group and Cauchy problems on white noise space are discussed.
\end{abstract}

\section{Introduction}

During the recent development of infinite dimensional analysis much attention has been paid to roles of differential operators, in particular, of infinite dimensional Laplacians. Among many variants of Laplacians on an infinite dimensional space, the Gross Laplacian, the number operator (or the Beltrami Laplacian) and the Lévy Laplacian have been studied extensively. Based on an abstract Wiener space, Gross [9] introduced an infinite dimensional Laplacian (presently called the Gross Laplacian) and studied differential equations in infinite dimension. The number operator being a central object in quantum physics, the associated Cauchy problem was discussed by Piech [22], [23]. It is also noteworthy from harmonic analysis that the number operator appears as a limit of the spherical Laplacians as the dimension tends to the infinity [24], [25]. As for the Lévy Laplacian [17] a series of new interesting features has been investigated recently [1], it is still somehow beyond our discussion of this paper.

It was Kuo [13] who first made an attempt to understand these Laplacians in a unified manner, namely, as operators acting on white noise functions [10]. Later on it was proved that the Gross Laplacian $\Delta_{G}$ and the number operator $N$ are in essence the only operators which are rotation-

\footnotetext{
Received June 25, 1996.

${ }^{1}$ Research supported by BSRI 95-1412.

${ }^{2}$ Research supported in part by Grant-in-Aid for Scientific Research No. 08640192, Ministry of Education, Japan
} 
invariant [18]. On the other hand, a linear combination of $\Delta_{G}$ and $N$ occurs as an infinitesimal generator of the Kuo-Fourier-Mehler transform [14], [15], and various one-parameter transformation groups are discussed [4], [11], see also [16]. White noise approach to infinite dimensional differential equations is also very interesting to study: the Cauchy problems associated with a certain generalized Gross Laplacian is discussed in [8] and those problems associated with $a N+b \Delta_{G}$ and their powers in the recent papers [4], [5]. Moreover, these Laplacians have been also studied toward de Rham-Hodge-Kodaira theory in infinite dimension, see e.g., [2], [7].

Using the standard notation in white noise calculus [12], [16], [19], the Gross Laplacian and the number operator are respectively expressed as

$$
\Delta_{G}=\int_{T} a_{t}^{2} d t, \quad N=\int_{T} a_{t}^{*} a_{t} d t
$$

where $a_{t}$ and $a_{t}^{*}$ are respectively the annihilation and creation operators at a point $t \in T, T$ being a certain parameter space but later taken to be $T=\mathbf{R}$. Since $a_{t}$ is a directional derivative (see (2.1)), the above operators are viewed as infinite dimensional (or to be more precise, continuous) analogues of finite dimensional Laplacians:

$$
\sum_{k=1}^{n}\left(\frac{d}{d x_{k}}\right)^{2}, \quad \sum_{k=1}^{n}\left(\frac{d}{d x_{k}}\right)^{*}\left(\frac{d}{d x_{k}}\right) .
$$

These operators are identical up to \pm 1 on the Euclidean space with Lebesgue measure; however, their infinite dimensional analogues $\Delta_{G}$ and $N$ are known to be completely different from each other. The purpose of this paper is to study their generalizations:

$$
N(f)=\int_{T} f(t) a_{t}^{*} a_{t} d t, \quad \Delta_{G}(g)=\int_{T} g(t) a_{t}^{2} d t
$$

which are referred to as second order differential operators of diagonal type. Obviously, $N(1)=N$ and $\Delta_{G}(1)=\Delta_{G}$. It is interesting to construct oneparameter transformation groups of which infinitesimal generator is $N(f)+$ $\Delta_{G}(g)$. In fact, employing the idea of an equicontinuous generator [20], [21], we shall discuss such a question in terms of power series expansion. The difficulty appearing here does not occur in the case of bounded operators on a Banach space but is typical for a nuclear Fréchet space.

The paper is organized as follows: In Section 1 we assemble standard notations used in white noise calculus and in Section 2 we make a quick 
review of an integral kernel operator which is a central concept in the operator theory on white noise functions, for further details see [19]. In Section 3 we introduce second order differential operators of diagonal type. Section 4 surveys some basic properties of an equicontinuous generator and contains a criterion in the white noise case. In Section 5 we investigate conditions for second order differential operators of diagonal type to be equicontinuous. We then discuss some applications. Section 6 is devoted to a study of representation of an infinite dimensional analogue of $a x+b$ group and Section 7 discusses the associated Cauchy problems.

Acknowledgements. The present joint work was started during the third named author's stay at Sogang University in November 1995. He is most grateful to the Department of Mathematics, in particular to Professor D. M. Chung for their kind hospitality.

\section{$\S 1$. White noise functions}

The whole discussion is based on the special choice of a real Gelfand triple:

$$
E \equiv \mathcal{S}(\mathbf{R}) \subset H \equiv L^{2}(\mathbf{R}, d t) \subset E^{*} \equiv \mathcal{S}^{\prime}(\mathbf{R})
$$

However, $\mathbf{R}$ can be replaced with $\mathbf{R}^{n}$ with no essential change (sometimes more interesting for applications); furthermore, a more axiomatic approach $[12],[19]$ is also possible with simple modification. It is noteworthy that the Gelfand triple (1.1) is constructed from the differential operator $A=$ $1+t^{2}-d^{2} / d t^{2}$. In fact, $E=\mathcal{S}(\mathbf{R})$ is identified (up to null functions) with the space of functions $\xi \in H$ such that $|\xi|_{p} \equiv\left|A^{p} \xi\right|_{0}<\infty$ for any $p \in \mathbf{R}$, where $|\cdot|_{0}$ stands for the norm of $H$, and the topology of $E$ is given by the norms $|\cdot|_{p}, p \in \mathbf{R}$. Since $A$ is a positive selfadjoint operator with Hilbert-Schmidt inverse, $E$ becomes a countable Hilbert nuclear space. By definition $E^{*}$ is the strong dual space of $E$. The canonical bilinear form on $E^{*} \times E$ and the real inner product of $H$ are denoted by the same symbol $\langle\cdot, \cdot\rangle$ because they are consistent.

The Gaussian measure $\mu$ is by definition a unique probability measure on $E^{*}$ of which characteristic function is

$$
\exp \left(-\frac{1}{2}|\xi|_{0}^{2}\right)=\int_{E^{*}} e^{i\langle x, \xi\rangle} \mu(d x), \quad \xi \in E
$$


The probability space $\left(E^{*}, \mu\right)$ is called the white noise space or the Gaussian space. With each $\xi \in E_{\mathbf{C}}$ we associate a function on $E^{*}$ defined by

$$
\phi_{\xi}(x)=\exp \left(\langle x, \xi\rangle-\frac{1}{2}\langle\xi, \xi\rangle\right), \quad x \in E^{*},
$$

which is called an exponential vector. In particular, $\phi_{0}$ is called the vacuum vector. The correspondence

$$
\phi_{\xi} \longleftrightarrow\left(1, \frac{\xi}{1 !}, \frac{\xi^{\otimes 2}}{2 !}, \frac{\xi^{\otimes 3}}{3 !}, \cdots\right), \quad \xi \in E_{\mathbf{C}}
$$

is uniquely extended to a unitary isomorphism between $L^{2}\left(E^{*}, \mu\right)$ and the Boson Fock space over $H_{\mathbf{C}}$, denoted by $\Gamma\left(H_{\mathbf{C}}\right)$, which is the celebrated Wiener-Itô-Segal isomorphism. If $\phi \in L^{2}\left(E^{*}, \mu\right)$ and $\left(f_{n}\right) \in \Gamma\left(H_{\mathbf{C}}\right)$ are related, we write $\phi \sim\left(f_{n}\right)$ simply. In that case,

$$
\|\phi\|_{0}^{2} \equiv \int_{E^{*}}|\phi(x)|^{2} \mu(d x)=\sum_{n=0}^{\infty} n !\left|f_{n}\right|_{0}^{2} .
$$

For any $p \in \mathbf{R}$ we put

$$
\|\phi\|_{p}^{2}=\sum_{n=0}^{\infty} n !\left|f_{n}\right|_{p}^{2}=\sum_{n=0}^{\infty} n !\left|\left(A^{\otimes n}\right)^{p} f_{n}\right|_{0}^{2}, \quad \phi \sim\left(f_{n}\right) .
$$

Let $(E)$ be the subspace of functions $\phi \in L^{2}\left(E^{*}, \mu\right)$ such that $\|\phi\|_{p}<\infty$ for all $p$. Then $(E)$ becomes a nuclear Fréchet space with the defining seminorms $\|\cdot\|_{p}, p \in \mathbf{R}$. the dual space $(E)^{*}$ consists of all elements $\Phi \sim$ $\left(F_{n}\right)$ such that $F_{n} \in\left(E_{\mathbf{C}}^{\otimes n}\right)_{\text {sym }}^{*}$ and $\|\Phi\|_{-p}<\infty$ for some $p \geq 0$. We thereby obtain a complex Gelfand triple:

$$
(E) \subset L^{2}\left(E^{*}, \mu\right) \subset(E)^{*} .
$$

Elements in $(E)$ and $(E)^{*}$ are called a test (white noise) function and a generalized (white noise) function, respectively. We denote by $\langle\langle\cdot, \cdot\rangle\rangle$ the canonical bilinear form on $(E)^{*} \times(E)$. Then we have

$$
\langle\langle\Phi, \phi\rangle\rangle=\sum_{n=0}^{\infty} n !\left\langle F_{n}, f_{n}\right\rangle, \quad \Phi \sim\left(F_{n}\right) \in(E)^{*}, \quad \phi \sim\left(f_{n}\right) \in(E),
$$

where the canonical bilinear form on $\left(E_{\mathbf{C}}^{\otimes n}\right)^{*} \times\left(E_{\mathbf{C}}^{\otimes n}\right)$ is denoted also by $\langle\cdot, \cdot\rangle$. 


\section{§. Integral kernel operators}

Based on the Gelfand triple (1.4) Fock space operators have been studied in detail [19], where a central role is played by integral kernel operators. Unless otherwise specified, $(E)^{*}$ is equipped with the strong dual topology. Let $\mathcal{L}\left((E),(E)^{*}\right)$ and $\mathcal{L}((E),(E))$ denote the spaces of continuous operators from $(E)$ into $(E)^{*}$ and those from $(E)$ into itself, respectively. These spaces are equipped with the topology of uniform convergence on every bounded subset. Then the natural inclusion $\mathcal{L}((E),(E)) \rightarrow \mathcal{L}\left((E),(E)^{*}\right)$ is continuous, and by adjoint $\mathcal{L}((E),(E))$ and $\mathcal{L}\left((E)^{*},(E)^{*}\right)$ are isomorphic.

With each $y \in E_{\mathbf{C}}^{*}$ we may associate an annihilation operator $D_{y} \in$ $\mathcal{L}((E),(E))$ which is uniquely determined by $D_{y} \phi_{\xi}=\langle y, \xi\rangle \phi_{\xi}, \xi \in E_{\mathbf{C}}$. Since $\delta_{t} \in E^{*}$ for any $t \in \mathbf{R}$,

$$
a_{t}=D_{\delta_{t}}, \quad t \in \mathbf{R}
$$

belongs to $\mathcal{L}((E),(E))$. This is called the annihilation operator at a point $t \in \mathbf{R}$. In some literatures $a_{t}$ is also denoted by $\partial_{t}$ and is called Hida's differential operator. It is known that $a_{t}$ is a differential operator along the direction $\delta_{t}$, namely,

$$
a_{t} \phi(x)=\lim _{\theta \rightarrow 0} \frac{\phi\left(x+\theta \delta_{t}\right)-\phi(x)}{\theta}, \quad \phi \in(E), \quad t \in \mathbf{R}, \quad x \in E^{*} .
$$

The creation operator at a point is by definition the adjoint $a_{t}^{*} \in \mathcal{L}\left((E)^{*}\right.$, $\left.(E)^{*}\right)$. We have then the so-called canonical commutation relation:

$$
\left[a_{s}, a_{t}\right]=0, \quad\left[a_{s}^{*}, a_{t}^{*}\right]=0, \quad\left[a_{s}, a_{t}^{*}\right]=\delta_{s}(t) I, \quad s, t \in \mathbf{R},
$$

where the last relation is understood in a generalized sense.

For any $\kappa \in\left(E_{\mathbf{C}}^{\otimes(l+m)}\right)^{*}$ there exists an operator $\Xi_{l, m}(\kappa) \in \mathcal{L}\left((E),(E)^{*}\right)$ uniquely determined by

$$
\left\langle\left\langle\Xi_{l, m}(\kappa) \phi_{\xi}, \phi_{\eta}\right\rangle\right\rangle=\left\langle\kappa, \eta^{\otimes l} \otimes \xi^{\otimes m}\right\rangle e^{\langle\xi, \eta\rangle}, \quad \xi, \eta \in E_{\mathbf{C}} .
$$

In that case we also employ a formal integral expression:

$$
\begin{aligned}
& \Xi_{l, m}(\kappa) \\
= & \int_{\mathbf{R}^{l+m}} \kappa\left(s_{1}, \cdots, s_{l}, t_{1}, \cdots, t_{m}\right) a_{s_{1}}^{*} \cdots a_{s_{l}}^{*} a_{t_{1}} \cdots a_{t_{m}} d s_{1} \cdots d s_{l} d t_{1} \cdots d t_{m} .
\end{aligned}
$$


We call $\Xi_{l, m}(\kappa)$ an integral kernel operator with kernel distribution $\kappa$. The kernel distribution is uniquely determined whenever taken from the subspace

$$
\left(E_{\mathbf{C}}^{\otimes(l+m)}\right)_{\text {sym }(l, m)}^{*}=\left\{\kappa \in\left(E_{\mathbf{C}}^{\otimes(l+m)}\right)^{*} ; s_{l, m}(\kappa)=\kappa\right\},
$$

where $s_{l, m}$ is the symmetrizing operator with respect to the first $l$ and the last $m$ variables independently. By definition $\Xi_{0,0}(\kappa)=\kappa I$ is a scalar operator.

Integral kernel operators in $\mathcal{L}((E),(E))$ will be particularly important in the operator theory on white noise functions, see also [19]. Recall the canonical isomorphism $\left(E_{\mathbf{C}}^{\otimes l}\right) \otimes\left(E_{\mathbf{C}}^{\otimes m}\right)^{*} \cong \mathcal{L}\left(E_{\mathbf{C}}^{\otimes m}, E_{\mathbf{C}}^{\otimes l}\right)$, which follows from the kernel theorem.

LEMMA 2.1. Let $\kappa \in\left(E_{\mathbf{C}}^{\otimes(l+m)}\right)^{*}$. Then $\Xi_{l, m}(\kappa) \in \mathcal{L}((E),(E))$ if and only if $\kappa \in\left(E_{\mathbf{C}}^{\otimes l}\right) \otimes\left(E_{\mathbf{C}}^{\otimes m}\right)^{*}$. In particular, $\Xi_{0, m}(\kappa) \in \mathcal{L}((E),(E))$ for any $\kappa \in\left(E_{\mathbf{C}}^{\otimes m}\right)^{*}$.

\section{$\S 3$. Second order differential operators of diagonal type}

Let $\tau \in\left(E_{\mathbf{C}} \otimes E_{\mathbf{C}}\right)^{*}$ be defined by

$$
\langle\tau, \eta \otimes \xi\rangle=\langle\xi, \eta\rangle, \quad \xi, \eta \in E_{\mathbf{C}} .
$$

In fact, $\tau \in E_{\mathbf{C}} \otimes E_{\mathbf{C}}^{*}$ since $\tau$ corresponds to the identity operator under the canonical isomorphism $E_{\mathbf{C}} \otimes E_{\mathbf{C}}^{*} \cong \mathcal{L}\left(E_{\mathbf{C}}, E_{\mathbf{C}}\right)$. Thus by Lemma 2.1,

$$
N=\Xi_{1,1}(\tau)=\int_{\mathbf{R}^{2}} \tau(s, t) a_{s}^{*} a_{t} d s d t\left(=\int_{\mathbf{R}} a_{t}^{*} a_{t} d t \quad \text { for simplicity }\right)
$$

belongs to $\mathcal{L}((E),(E))$. This is called the number operator. On the other hand,

$$
\Delta_{G}=\Xi_{0,2}(\tau)=\int_{\mathbf{R}^{2}} \tau\left(t_{1}, t_{2}\right) a_{t_{1}} a_{t_{2}} d t_{1} d t_{2}\left(=\int_{\mathbf{R}} a_{t}^{2} d t \quad \text { for simplicity }\right)
$$

also belongs to $\mathcal{L}((E),(E))$ and is called the Gross Laplacian. Having some typical properties of a finite dimensional Laplacian, $N$ and $\Delta_{G}$ are considered as natural infinite dimensional (or continuous) analogues of a finite dimensional Laplacian [18], [19].

To introduce their generalizations we need notation. Let $\mathcal{M}\left(E_{\mathbf{C}}, E_{\mathbf{C}}^{*}\right)$ be the space of $\mathbf{C}$-valued measurable functions $f$ on $\mathbf{R}$ such that the multiplication operator $M_{f}$ defined as $M_{f} \xi=f \xi, \xi \in E_{\mathbf{C}}$, belongs to $\mathcal{L}\left(E_{\mathbf{C}}, E_{\mathbf{C}}^{*}\right)$. 
We identify $\mathcal{M}\left(E_{\mathbf{C}}, E_{\mathbf{C}}^{*}\right)$ with a subspace of $\mathcal{L}\left(E_{\mathbf{C}}, E_{\mathbf{C}}^{*}\right)$ by the injection $f \mapsto M_{f}$. Put

$$
\mathcal{M}\left(E_{\mathbf{C}}, E_{\mathbf{C}}\right)=\mathcal{M}\left(E_{\mathbf{C}}, E_{\mathbf{C}}^{*}\right) \cap \mathcal{L}\left(E_{\mathbf{C}}, E_{\mathbf{C}}\right)
$$

LEMMA 3.1. (1) $M_{f}^{*}=M_{f}$ for any $f \in \mathcal{M}\left(E_{\mathbf{C}}, E_{\mathbf{C}}^{*}\right)$.

(2) If $f \in \mathcal{M}\left(E_{\mathbf{C}}, E_{\mathbf{C}}\right), M_{f}$ is continuously extended to an operator from $E_{\mathbf{C}}^{*}$ into $E_{\mathbf{C}}^{*}$.

(3) $\mathcal{M}\left(E_{\mathbf{C}}, E_{\mathbf{C}}\right)$ is an abelian subalgebra of $\mathcal{L}\left(E_{\mathbf{C}}, E_{\mathbf{C}}\right)$.

The proofs are straightforward. For $f \in \mathcal{M}\left(E_{\mathbf{C}}, E_{\mathbf{C}}^{*}\right)$ we denote by $\tau(f) \in\left(E_{\mathbf{C}} \otimes E_{\mathbf{C}}\right)^{*}$ the distribution uniquely determined by

$$
\langle\tau(f), \eta \otimes \xi\rangle=\left\langle M_{f} \xi, \eta\right\rangle, \quad \xi, \eta \in E_{\mathbf{C}}
$$

In other words, the relation between $M_{f}$ and $\tau(f)$ follows from the canonical isomorphism $\mathcal{L}\left(E_{\mathbf{C}}, E_{\mathbf{C}}^{*}\right) \cong\left(E_{\mathbf{C}} \otimes E_{\mathbf{C}}\right)^{*}$. It is obvious that $I=M_{1} \in$ $\mathcal{M}\left(E_{\mathbf{C}}, E_{\mathbf{C}}\right)$. In that case $\tau(1)=\tau$, see (3.1) and (3.2). We understand that $\tau(f)$ is a distribution concentrated on the diagonal of $\mathbf{R} \times \mathbf{R}$. Now for $f \in \mathcal{M}\left(E_{\mathbf{C}}, E_{\mathbf{C}}^{*}\right)$ we define

$$
\begin{aligned}
& \Delta_{G}(f)=\Xi_{0,2}(\tau(f))=\int_{\mathbf{R}} f(t) a_{t}^{2} d t, \\
& N(f)=\Xi_{1,1}(\tau(f))=\int_{\mathbf{R}} f(t) a_{t}^{*} a_{t} d t .
\end{aligned}
$$

These are called generally second order differential operators of diagonal type. Note also that $\Delta_{G}(f)$ and $N(f)$ are obtained by smearing the Gross Laplacian density $a_{t}^{2} \in \mathcal{L}((E),(E))$ and the number density $a_{t}^{*} a_{t} \in \mathcal{L}((E)$, $\left.(E)^{*}\right)$, respectively.

By a general theory of integral kernel operators (see $\S 2$ ) one can immediately deduce the following

Proposition 3.2. If $f \in \mathcal{M}\left(E_{\mathbf{C}}, E_{\mathbf{C}}^{*}\right)$, then $\Delta_{G}(f) \in \mathcal{L}((E),(E))$ and $N(f) \in \mathcal{L}\left((E),(E)^{*}\right)$. In particular, $\Delta_{G}(1)=\Delta_{G}$ and $N(1)=N$. Moreover, $N(f) \in \mathcal{L}((E),(E))$ if and only if $\tau(f) \in E_{\mathbf{C}} \otimes E_{\mathbf{C}}^{*}$, i.e., if and only if $f \in \mathcal{M}\left(E_{\mathbf{C}}, E_{\mathbf{C}}\right)$. 


\section{§4. Equicontinuous generators}

Being motivated by the standard terminology [26, Chapter IX], we have introduced in [20] the concept of an equicontinuous generator to construct one-parameter subgroups in $G L((E))$, and in [21] we have discussed it in a more general situation. Here we recall the generality. For the moment let $\mathfrak{X}$ be a barreled locally convex Hausdorff space (what we need here is the Banach-Steinhaus theorem) with defining seminorms $\left\{\|\cdot\|_{\alpha}\right\}_{\alpha}$ which are assumed to be directed without loss of generality.

Definition 4.1. A one-parameter subgroup $\left\{\Omega_{z}\right\}_{z \in \mathbf{C}} \subset G L(\mathfrak{X})$ is called holomorphic if there exists an operator $\Xi \in \mathcal{L}(\mathfrak{X}, \mathfrak{X})$ such that

$$
\lim _{z \rightarrow 0}\left\|\frac{\Omega_{z} \phi-\phi}{z}-\Xi \phi\right\|_{\alpha}=0 \quad \text { for all } \phi \in \mathfrak{X} \text { and } \alpha .
$$

In that case $\Xi$ is unique and is called the infinitesimal generator of $\left\{\Omega_{z}\right\}$.

If $\left\{\Omega_{z}\right\}_{z \in \mathbf{C}} \subset G L(\mathfrak{X})$ is a holomorphic one-parameter subgroup, for any $\Phi \in \mathfrak{X}^{*}$ and $\phi \in \mathfrak{X}$ the complex function $z \mapsto\left\langle\left\langle\Phi, \Omega_{z} \phi\right\rangle\right\rangle$ is entire holomorphic. Moreover, as is easily seen,

$$
\frac{d}{d z}\left\langle\left\langle\Phi, \Omega_{z} \phi\right\rangle\right\rangle=\left\langle\left\langle\Phi, \Omega_{z} \Xi \phi\right\rangle\right\rangle=\left\langle\left\langle\Phi, \Xi \Omega_{z} \phi\right\rangle\right\rangle, \quad \Phi \in \mathfrak{X}^{*}, \quad \phi \in \mathfrak{X} .
$$

In particular, note that $\left[\Xi, \Omega_{z}\right]=0$.

Lemma 4.2. ([21]) For $\Xi \in \mathcal{L}(\mathfrak{X}, \mathfrak{X})$ the following four conditions are equivalent:

(i) there exists some $R>0$ such that $\left\{(R \Xi)^{n} / n ! ; n=0,1,2, \cdots\right\}$ is equicontinuous;

(ii) $\left\{(R \Xi)^{n} / n ! ; n=0,1,2, \cdots\right\}$ is equicontinuous for any $R>0$;

(iii) $\Xi$ is the infinitesimal generator of some holomorphic one-parameter subgroup $\left\{\Omega_{z}\right\}_{z \in \mathbf{C}} \subset G L(\mathfrak{X})$ such that $\left\{\Omega_{z} ;|z|<R\right\}$ is equicontinuous for some $R>0$.

(iv) $\Xi$ is the infinitesimal generator of some holomorphic one-parameter subgroup $\left\{\Omega_{z}\right\}_{z \in \mathbf{C}} \subset G L(\mathfrak{X})$ such that $\left\{\Omega_{z} ;|z|<R\right\}$ is equicontinuous for any $R>0$. 
Definition 4.3. An operator $\Xi \in \mathcal{L}(\mathfrak{X}, \mathfrak{X})$ satisfying the condition mentioned in Lemma 4.2 is called an equicontinuous generator.

If $\Xi \in \mathcal{L}(\mathfrak{X}, \mathfrak{X})$ is an equicontinuous generator, the corresponding holomorphic one-parameter subgroup of $G L(\mathfrak{X})$ is also denoted by $\{\exp (z \Xi)\}_{z \in \mathbf{C}}$. It is known that

$$
\exp (z \Xi)=\sum_{n=0}^{\infty} \frac{z^{n}}{n !} \Xi^{n}, \quad z \in \mathbf{C}
$$

where the right hand side converges in $\mathcal{L}(\mathfrak{X}, \mathfrak{X})$ with respect to the bounded convergence topology.

In this paper we are mostly devoted to the case of $\mathfrak{X}=(E)$. In that case the characterization theorem of an operator symbol $[19, \S 4.4]$ is particularly useful.

Proposition 4.4. Let $\Xi \in \mathcal{L}((E),(E))$ and $\left\{\Omega_{z}\right\}_{z \in \mathbf{C}} \subset G L((E))$ be a one-parameter subgroup. Assume that for any $p \geq 0$ and $\epsilon>0$ there exist $q \geq 0$ and a function $C(z)$ defined on $\mathbf{C}$ with $\lim _{z \rightarrow 0} C(z)=0$ such that

$$
\left|\left\langle\left\langle\left(\frac{\Omega_{z}-I}{z}-\Xi\right) \phi_{\xi}, \phi_{\eta}\right\rangle\right\rangle\right| \leq C(z) \exp \epsilon\left(|\xi|_{p+q}^{2}+|\eta|_{-p}^{2}\right), \quad \xi, \eta \in E_{\mathbf{C}} .
$$

Then $\left\{\Omega_{z}\right\}$ is a holomorphic one-parameter subgroup of $G L((E))$. If in addition $C(z)$ is bounded on every compact subset of $\mathbf{C}$, then $\Xi$ is an equicontinuous generator.

Proof. For simplicity we put

$$
\Xi_{z}=\frac{\Omega_{z}-I}{z}-\Xi, \quad z \in \mathbf{C}, \quad z \neq 0 .
$$

Then by the assumption we have

$$
\left|\left\langle\left\langle\Xi_{z} \phi_{\xi}, \phi_{\eta}\right\rangle\right\rangle\right| \leq C(z) \exp \epsilon\left(|\xi|_{p+q}^{2}+|\eta|_{-p}^{2}\right), \quad \xi, \eta \in E_{\mathbf{C}}
$$

Applying a general result [19, Corollary 4.4.11], we obtain

$$
\left\|\Xi_{z} \phi\right\|_{p-1} \leq C(z) M(\epsilon, q, r)\|\phi\|_{p+q+r+1}, \quad \phi \in(E),
$$


where $M(\epsilon, q, r)$ is a constant determined for $\epsilon<\left(2 e^{3} \delta^{2}\right)^{-1}, r \geq r_{0}(q) \geq 0$. Hence $\left\{\Omega_{z}\right\}$ is a holomorphic one-parameter group. Moreover, from (4.3) one can deduce

$$
\begin{aligned}
\left\|\Omega_{z} \phi\right\|_{p-1} \leq\|\phi\|_{p-1}+|z| & \|\Xi \phi\|_{p-1} \\
& +|z| C(z) M(\epsilon, q, r)\|\phi\|_{p+q+r+1}
\end{aligned}
$$

Since $\Xi \in \mathcal{L}((E),(E))$, we may choose $C \geq 0$ and $s \geq q+r+1$ such that

$$
\|\Xi \phi\|_{p-1} \leq C\|\phi\|_{p+s}, \quad \phi \in(E) .
$$

Then (4.4) becomes

$$
\begin{aligned}
\left\|\Omega_{z} \phi\right\|_{p-1} & \leq\|\phi\|_{p+s}+C|z|\|\phi\|_{p+s}+|z| C(z) M(\epsilon, q, r)\|\phi\|_{p+s} \\
& =(1+C|z|+|z| C(z) M(\epsilon, q, r))\|\phi\|_{p+s} .
\end{aligned}
$$

Therefore, if $C(z)$ is bounded on every compact subset of $\mathbf{C}$, the above inequality implies that $\left\{\Omega_{z} ;|z|<R\right\}$ is equicontinuous for any $R>0$. Hence by definition $\Xi$ is an equicontinuous generator.

During the above proof we have established the following result which is also useful.

COROllary 4.5. Let $\left\{\Xi_{i}\right\}_{i \in I}$ be a subset of $\mathcal{L}((E),(E))$. If for any $\epsilon>0$ and $p \geq 0$ there exist $q \geq 0$ and $C \geq 0$ such that

$$
\left|\left\langle\left\langle\Xi_{i} \phi_{\xi}, \phi_{\eta}\right\rangle\right\rangle\right| \leq C \exp \epsilon\left(|\xi|_{p+q}^{2}+|\eta|_{-p}^{2}\right), \quad \xi, \eta \in E_{\mathbf{C}}, \quad i \in I
$$

then the family $\left\{\Xi_{i}\right\}_{i \in I}$ is equicontinuous.

\section{$\S 5$. Equicontinuity}

We first prove the following general result.

Lemma 5.1. For any $\kappa \in\left(E_{\mathbf{C}} \otimes E_{\mathbf{C}}\right)^{*}$,

$$
\Xi_{0,2}(\kappa)=\int_{\mathbf{R}^{2}} \kappa\left(t_{1}, t_{2}\right) a_{t_{1}} a_{t_{2}} d t_{1} d t_{2}
$$

is an equicontinuous generator. 
Proof. For simplicity we put $\Xi=\Xi_{0,2}(\kappa)$. Suppose $p \geq 0$ and $q>0$. Then direct application of [19, Theorem 4.3.9] leads us to

$$
\left\|\Xi^{n} \phi\right\|_{p} \leq \rho^{-q / 2}(2 n)^{n}\left(\frac{\rho^{-q}}{-2 e q \log \rho}\right)^{n}|\kappa|_{-(p+q)}^{n}\|\phi\|_{p+q},
$$

where $\rho=\inf \operatorname{Spec}(A)=1 / 2$. By $|\kappa|_{-(p+q)} \leq \rho^{2 q}|\kappa|_{-p}$ we obtain

$$
\left\|\Xi^{n} \phi\right\|_{p} \leq \rho^{-q / 2}(2 n)^{n}\left(\frac{\rho^{q}}{-2 e q \log \rho}\right)^{n}|\kappa|_{-p}^{n}\|\phi\|_{p+q},
$$

where $\phi \in(E)$ and $n=0,1,2, \cdots$. In view of the simple identity

$$
\frac{(2 n)^{n}}{n !}|\kappa|_{-p}^{n}\left(\frac{\rho^{q}}{-2 e q \log \rho}\right)^{n}=\frac{n^{n} \sqrt{n}}{n ! e^{n}} \times \frac{1}{\sqrt{n}} \times\left(\frac{\rho^{q}|\kappa|_{-p}}{-q \log \rho}\right)^{n}
$$

for any $p \geq 0$ we choose $q>0$ such that $\rho^{q}|\kappa|_{-p} \leq-q \log \rho$. It then follows from the Stirling formula that

$$
M=\sup _{n} \frac{(2 n)^{n}}{n !}|\kappa|_{-p}^{n}\left(\frac{\rho^{q}}{-2 e q \log \rho}\right)^{n}<\infty .
$$

Hence (5.1) becomes

$$
\left\|\frac{1}{n !} \Xi^{n} \phi\right\|_{p} \leq \rho^{-q / 2} M\|\phi\|_{p+q}, \quad \phi \in(E) .
$$

Namely, $\left\{\Xi^{n} / n ! ; n=0,1,2, \cdots\right\}$ is equicontinuous. Consequently, we see from Lemma 4.2 that $\Xi=\Xi_{0,2}(\kappa)$ is an equicontinuous generator.

Theorem 5.2. For any $f \in \mathcal{M}\left(E_{\mathbf{C}}, E_{\mathbf{C}}^{*}\right)$,

$$
\Delta_{G}(f)=\Xi_{0,2}(\tau(f))=\int_{\mathbf{R}} f(t) a_{t}^{2} d t
$$

is an equicontinuous generator.

Proof. Recall that $\tau(f) \in\left(E_{\mathbf{C}} \otimes E_{\mathbf{C}}\right)^{*}$ whenever $f \in \mathcal{M}\left(E_{\mathbf{C}}, E_{\mathbf{C}}^{*}\right)$. The assertion is then immediate from Lemma 5.1.

For $K \in \mathcal{L}\left(E_{\mathbf{C}}, E_{\mathbf{C}}\right)$ we define $\Gamma(K), d \Gamma(K) \in \mathcal{L}((E),(E))$ by

$$
\Gamma(K) \phi \sim\left(K^{\otimes n} f_{n}\right), \quad d \Gamma(K) \phi \sim\left(n K \otimes I^{\otimes(n-1)} f_{n}\right),
$$


where $\phi \sim\left(f_{n}\right) \in(E)$. If $K$ and $\kappa \in E_{\mathbf{C}} \otimes E_{\mathbf{C}}^{*}$ are related by the kernel theorem, i.e.,

$$
\langle K \xi, \eta\rangle=\langle\kappa, \eta \otimes \xi\rangle, \quad \xi, \eta \in E_{\mathbf{C}}
$$

then $d \Gamma(K)=\Xi_{1,1}(\kappa)$.

LEMMA 5.3. Let $\left\{S_{z}\right\}_{z \in \mathbf{C}} \subset G L\left(E_{\mathbf{C}}\right)$ be a holomorphic one-parameter subgroup with an equicontinuous generator $K \in \mathcal{L}\left(E_{\mathbf{C}}, E_{\mathbf{C}}\right)$. Then $\left\{\Gamma\left(S_{z}\right)\right\}_{z \in \mathbf{C}}$ is a holomorphic one-parameter subgroup of $G L((E))$ with equicontinuous generator $d \Gamma(K)$. Conversely, if $d \Gamma(K)$ is an equicontinuous generator, then so is $K$.

Proof. The first part has appeared in [21]. As for the second part we need only to note that the action of $d \Gamma(K)$ on the one-particle space is the same as $K$, see (5.3).

Then the next is a simple consequence.

Lemma 5.4. Assume that $\kappa \in E_{\mathbf{C}} \otimes E_{\mathbf{C}}^{*}$ and $K \in \mathcal{L}\left(E_{\mathbf{C}}, E_{\mathbf{C}}\right)$ are related as in (5.4). Then the integral kernel operator

$$
\Xi_{1,1}(\kappa)=\int_{\mathbf{R}^{2}} \kappa(s, t) a_{s}^{*} a_{t} d s d t
$$

is an equicontinuous generator if and only if so is $K$.

It can be proved easily that if $|\kappa|_{1,1 ; p,-p} \equiv\left|\left(A^{p} \otimes A^{-p}\right) \kappa\right|_{0}<\infty$ for all $p \geq 0$, the corresponding operator $K \in \mathcal{L}\left(E_{\mathbf{C}}, E_{\mathbf{C}}\right)$ is an equicontinuous generator. This occurs, for example, when $\kappa$ is a finite linear combination of eigenvectors of $A \otimes A$. On the other hand, the case of $\kappa=\tau$ or equivalently of $K=I$ is included in Lemma 5.4. The following result generalizes this point, the proof of which follows immediately from Lemma 5.4.

THEOREM 5.5. Let $f \in \mathcal{M}\left(E_{\mathbf{C}}, E_{\mathbf{C}}\right)$. If $M_{f}$ is an equicontinuous generator, so is

$$
N(f)=\Xi_{1,1}(\tau(f))=\int_{\mathbf{R}} f(t) a_{t}^{*} a_{t} d t .
$$

As for a concrete example of an equicontinuous generator on $E=\mathcal{S}(\mathbf{R})$ we only mention the following results of which proofs are easy and omitted. 
LEMma 5.6. Let $f$ be a $C^{\infty}$-function on $\mathbf{R}$ whose derivatives $f^{(\alpha)}$ are slowly increasing, $\alpha=0,1,2, \cdots$. Then $f \in E_{\mathbf{C}}^{*}$ and $M_{f} \in \mathcal{L}\left(E_{\mathbf{C}}, E_{\mathbf{C}}\right)$, that is, $f \in \mathcal{M}\left(E_{\mathbf{C}}, E_{\mathbf{C}}\right)$.

LEMMA 5.7. Let $f$ be a $C^{\infty}$-function on $\mathbf{R}$. If every derivative $f^{(\alpha)}$ is bounded, the multiplication operator $M_{f} \in \mathcal{L}\left(E_{\mathbf{C}}, E_{\mathbf{C}}\right)$ is an equicontinuous generator.

Combining Theorem 5.5 and Lemma 5.7, we immediately obtain

Proposition 5.8. $N(f)$ is an equicontinuous generator for any $f \in$ $E_{\mathbf{C}}$.

\section{$\S 6$. An infinite dimensional analogue of $a x+b$ group}

We shall first prove the commutation relation among second order differential operators of diagonal type. Recall that $N(f) \in \mathcal{L}((E),(E))$ for any $f \in \mathcal{M}\left(E_{\mathbf{C}}, E_{\mathbf{C}}\right)$ and that $\Delta_{G}(g) \in \mathcal{L}((E),(E))$ for any $g \in \mathcal{M}\left(E_{\mathbf{C}}, E_{\mathbf{C}}^{*}\right)$.

TheOREM 6.1. Let $f_{1}, f_{2}, f \in \mathcal{M}\left(E_{\mathbf{C}}, E_{\mathbf{C}}\right)$ and $g_{1}, g_{2}, g \in \mathcal{M}\left(E_{\mathbf{C}}, E_{\mathbf{C}}^{*}\right)$. Then the following commutation relations holds:

$$
\begin{gathered}
{\left[N\left(f_{1}\right), N\left(f_{2}\right)\right]=0,} \\
{\left[\Delta_{G}\left(g_{1}\right), \Delta_{G}\left(g_{2}\right)\right]=0,} \\
{\left[N(f), \Delta_{G}(g)\right]=-2 \Delta_{G}(f g) .}
\end{gathered}
$$

Proof. In general, $\Xi_{0,2}(\kappa)$ with

$\kappa \in\left(E_{\mathbf{C}} \otimes E_{\mathbf{C}}\right)^{*}$ involves only annihilation operators, and so they commute each other, see also [20, Theorem 3.3]. Then (6.2) is a particular case. As for (6.3), since both sides are continuous operators, it is sufficient to check the identity for a function of the form $\phi \sim\left(0, \cdots, 0, \xi^{\otimes n}, 0, \cdots\right)$. The verification is a simple computation. The proof of (6.1) is given below.

Suppose $S_{1} \in \mathcal{L}\left(E_{\mathbf{C}}, E_{\mathbf{C}}\right)$ and $S_{2} \in \mathcal{L}\left(E_{\mathbf{C}}, E_{\mathbf{C}}^{*}\right)$. Let $f_{1} \in E_{\mathbf{C}} \otimes E_{\mathbf{C}}^{*}$ and $f_{2} \in\left(E_{\mathbf{C}} \otimes E_{\mathbf{C}}\right)^{*}$ be the corresponding elements, respectively, see (5.4). Then we denote by $f_{2} * f_{1}$ the element of $\left(E_{\mathbf{C}} \otimes E_{\mathbf{C}}\right)^{*}$ corresponding to $S_{2} S_{1} \in \mathcal{L}\left(E_{\mathbf{C}}, E_{\mathbf{C}}^{*}\right)$. It is noted that

$$
f_{2} * f_{1}(s, t)=\int_{\mathbf{R}} f_{2}(s, u) f_{1}(u, t) d u
$$

in a generalized sense. 
LEMma 6.2. The convolution $\left(f_{2}, f_{1}\right) \mapsto f_{2} * f_{1}$ gives a separately continuous bilinear map from $\left(E_{\mathbf{C}} \otimes E_{\mathbf{C}}\right)^{*} \times\left(E_{\mathbf{C}} \otimes E_{\mathbf{C}}^{*}\right)$ into $\left(E_{\mathbf{C}} \otimes E_{\mathbf{C}}\right)^{*}$, and from $\left(E_{\mathbf{C}} \otimes E_{\mathbf{C}}^{*}\right) \times\left(E_{\mathbf{C}} \otimes E_{\mathbf{C}}^{*}\right)$ into $E_{\mathbf{C}} \otimes E_{\mathbf{C}}^{*}$.

Proof. It is known that the composition of operators $\left(S_{2}, S_{1}\right) \mapsto S_{2} S_{1}$ gives a separately continuous bilinear map from $\mathcal{L}\left(E_{\mathbf{C}}, E_{\mathbf{C}}^{*}\right) \times \mathcal{L}\left(E_{\mathbf{C}}, E_{\mathbf{C}}\right)$ into $\mathcal{L}\left(E_{\mathbf{C}}, E_{\mathbf{C}}^{*}\right)$ and from $\mathcal{L}\left(E_{\mathbf{C}}, E_{\mathbf{C}}\right) \times \mathcal{L}\left(E_{\mathbf{C}}, E_{\mathbf{C}}\right)$ into $\mathcal{L}\left(E_{\mathbf{C}}, E_{\mathbf{C}}\right)$, which follows from the definition of the topology. Our assertion is then immediate from the kernel theorem.

LEMMA 6.3. It holds that

$$
\left[\Xi_{1,1}\left(f_{1}\right), \Xi_{1,1}\left(f_{2}\right)\right]=\Xi_{1,1}\left(f_{1} * f_{2}-f_{2} * f_{1}\right), \quad f_{1}, f_{2} \in E_{\mathbf{C}} \otimes E_{\mathbf{C}}^{*}
$$

Proof. Let $S \in \mathcal{L}\left(E_{\mathbf{C}}, E_{\mathbf{C}}\right)$ be the operator corresponding to $f$. To prove the assertion it is sufficient to show the identity for $\phi \sim\left(0, \cdots, 0, \xi_{1} \otimes\right.$ $\left.\cdots \otimes \xi_{n}, 0, \cdots\right)$. By a direct computation we see that

$$
\Xi_{1,1}(f) \phi \sim\left(0, \cdots, 0, \sum_{k=1}^{n} \xi_{1} \otimes \cdots \otimes \xi_{k-1} \otimes S \xi_{k} \otimes \cdots \otimes \xi_{n}, 0, \cdots\right) .
$$

The assertion follows by the repeated application.

Proof of (6.1). We see from Lemma 6.3 that

$$
[N(f), N(g)]=\left[\Xi_{1,1}(\tau(f)), \Xi_{1,1}(\tau(g))\right]=\Xi_{1,1}(\tau(f) * \tau(g)-\tau(g) * \tau(f)) .
$$

By definition $\tau(f) * \tau(g)$ and $\tau(g) * \tau(f)$ correspond respectively to $M_{f} M_{g}$ and $M_{g} M_{f}$ under the canonical isomorphism. Since $\mathcal{M}\left(E_{\mathbf{C}}, E_{\mathbf{C}}\right)$ is an abelian algebra, we have $\tau(f) * \tau(g)=\tau(g) * \tau(f)$, which completes the proof.

In view of Theorem 6.1 we obtain the commutation relation among transformations obtained by exponentiating $N(f)$ and $\Delta_{G}(g)$. By Theorem $5.2, \Delta_{G}(g)$ is an equicontinuous generator for any $g \in E_{\mathbf{C}}$. On the other hand, it follows from Theorem 5.5 that $N(f)$ is an equicontinuous generator for any $f \in E_{\mathbf{C}}$.

TheOREM 6.4. For any $f, g \in E_{\mathbf{C}}$ it holds that

$$
\begin{aligned}
\exp N(f) \cdot \Delta_{G}(g) \cdot \exp (-N(f)) & =\Delta_{G}\left(e^{-2 f} g\right), \\
\exp N(f) \exp \Delta_{G}(g) \exp (-N(f)) & =\exp \Delta_{G}\left(e^{-2 f} g\right) .
\end{aligned}
$$


Therefore

$$
\begin{aligned}
\exp N\left(f_{1}\right) \exp \Delta_{G}\left(g_{1}\right) \exp N\left(f_{2}\right) \exp \Delta_{G}\left(g_{2}\right) \\
=\exp N\left(f_{1}+f_{2}\right) \exp \Delta_{G}\left(e^{2 f_{2}} g_{1}+g_{2}\right) .
\end{aligned}
$$

Proof. Since $\left[N(f), \Delta_{G}(g)\right]=-2 \Delta_{G}(f g)$, we have

$$
\begin{aligned}
e^{N(f)} \Delta_{G}(g) e^{-N(f)} & \left.\left.=\sum_{n=0}^{\infty} \frac{z^{n}}{n !}[\underbrace{N(f),[N(f), \cdots[N(f)}_{n \text { times }}, \Delta_{G}(g)] \cdots\right]\right] \\
& =\sum_{n=0}^{\infty} \frac{z^{n}}{n !}(-2)^{n} \Delta_{G}\left(f^{n} g\right) \\
& =\Delta_{G}\left(e^{-2 f} g\right) .
\end{aligned}
$$

Then (6.5) follows immediately. Here we note that $e^{f} g \in E_{\mathbf{C}}$ for any $f, g \in E_{\mathbf{C}} \cdot\left(\right.$ In fact, $e^{f}-1 \in E_{\mathbf{C}}$.)

In [20] we discussed relation between the complex $a x+b$ group (of 2 dimension) and the Lie group generated by the Gross Laplacian $\Delta_{G}$ and the number operator $N$. We here discuss an infinite dimensional generalization. For $\left(f_{1}, g_{1}\right),\left(f_{2}, g_{2}\right) \in \mathcal{S}(\mathbf{R}) \times \mathcal{S}(\mathbf{R})=E \times E$ we define their product by

$$
\left(f_{1}, g_{1}\right) \cdot\left(f_{2}, g_{2}\right)=\left(f_{1}+f_{2}, e^{f_{2}} g_{1}+g_{2}\right)
$$

It is then easily verified that $E \times E$ becomes a topological group under the above multiplication rule, which we denote by $G$ from now on. It is a semidirect product of $E$ and $E$. The complexification is discussed in a parallel way. Let $\alpha \in \mathbf{C}$ be fixed. We put

$$
\pi_{\alpha}(f, g)=\exp \frac{1}{2} N(f) \cdot \exp \alpha \Delta_{G}(g), \quad f, g \in E .
$$

Proposition 6.5. $\pi_{\alpha}$ is a continuous representation of $G$ for any $\alpha \in$ C. If $\alpha \neq 0, \pi_{\alpha}$ is faithful. For $\alpha, \beta \in \mathbf{C}$ with $\alpha \beta \neq 0, \pi_{\alpha}$ and $\pi_{\beta}$ are conjugate in $G L((E))$. In particular, if $|\alpha|=|\beta|$, two representations $\pi_{\alpha}$ and $\pi_{\beta}$ are unitarily equivalent.

Proof. That $\pi_{\alpha}$ is a continuous representation of $G$ follows from (6.6). By a straightforward computation we obtain

$$
\left\langle\left\langle\pi_{\alpha}(2 f, g) \phi_{\xi}, \phi_{\eta}\right\rangle\right\rangle=e^{\alpha\langle g \xi, \xi\rangle+\langle f \xi, \eta\rangle}\left\langle\left\langle\phi_{\xi}, \phi_{\eta}\right\rangle\right\rangle, \quad \xi, \eta \in E_{\mathbf{C}} .
$$


Hence $\pi_{\alpha}(2 f, g)=I$ if and only if $e^{\alpha\langle g \xi, \xi\rangle+\langle f \xi, \eta\rangle}=1$ for any $\xi, \eta \in E_{\mathbf{C}}$, hence, if and only if

$$
\exp \left\{\alpha z^{2}\langle g \xi, \xi\rangle+z\langle f \xi, \eta\rangle\right\}=1, \quad z \in \mathbf{C}, \quad \xi, \eta \in E_{\mathbf{C}}
$$

Differentiating with respect to $z$, we obtain

$$
\alpha\langle g \xi, \xi\rangle=\langle f \xi, \eta\rangle=0, \quad \xi, \eta \in E_{\mathbf{C}}
$$

If $\alpha \neq 0$, then (6.7) is equivalent to $f=g=0$, that is, $\pi_{\alpha}$ is a faithful representation.

For $\alpha, \beta \in \mathbf{C}$ with $\alpha \beta \neq 0$ choose $\gamma \in \mathbf{C}$ such that $e^{2 \gamma} \alpha=\beta$. Then we see that

$$
e^{-\gamma N} \pi_{\alpha}(f, g) e^{\gamma N}=\pi_{\beta}(f, g) .
$$

In particular, if $|\alpha|=|\beta|$, the above $\gamma$ is a pure imaginary. Namely $e^{\gamma N}$ is a unitary operator on $L^{2}\left(E^{*}, \mu\right)$.

\section{$\S 7$. Cauchy problems}

Finally we consider a Cauchy problem associated with $N(f)+\Delta_{G}(g)$, i.e., given $\psi$, we study the initial value problem:

$$
\frac{\partial \phi}{\partial t}=\left(N(f)+\Delta_{G}(g)\right) \phi, \quad \phi(0, x)=\psi(x), \quad t \geq 0, \quad x \in E^{*} .
$$

More generally, given $\Xi \in \mathcal{L}((E),(E))$ we consider

$$
\frac{\partial \phi}{\partial t}=\Xi \phi, \quad \phi(0, x)=\psi(x), \quad t \geq 0, \quad x \in E^{*}
$$

If $\Xi$ is an equicontinuous generator and $\psi \in(E)$, the solution of $(7.2)$ is obtained by

$$
\phi(t, x)=\exp (t \Xi) \psi=\sum_{n=0}^{\infty} \frac{t^{n}}{n !} \Xi^{n} \psi,
$$

where the right hand side converges in $(E)$. Moreover, $\phi(t, x)$ is extended to $\mathbf{C} \times E^{*}$ and becomes holomorphic in $t \in \mathbf{C}$. Therefore we do not need to distinguish equations of heat type and Schrödinger type. Thus, if $N(f)+\Delta_{G}(g)$ is an equicontinuous generator, the solution of (7.1) is immediately obtained. For example, Theorems 5.2 and 5.5 describe the condition for $\Delta_{G}(g)$ and $N(f)$ being equicontinuous generators. For their linear combination the situation becomes much more complicated. 
Proposition 7.1. Let $f \in \mathcal{M}\left(E_{\mathbf{C}}, E_{\mathbf{C}}\right)$ and $g \in \mathcal{M}\left(E_{\mathbf{C}}, E_{\mathbf{C}}^{*}\right) . \quad A s-$ sume that $M_{f}$ is an equicontinuous generator. If $f g=\alpha g$ for some $\alpha \in \mathbf{C}$, then $N(f)+\Delta_{G}(g)$ is an equicontinuous generator.

Proof. It follows from a general theory established in [21] that $N(f)+$ $\Delta_{G}(g)$ is an equicontinuous generator if so are both $N(f)$ and $\Delta_{G}(g)$ and if

$$
\left[N(f), \Delta_{G}(g)\right]=\alpha \Delta_{G}(g) \quad \text { for some } \alpha \in \mathbf{C} .
$$

In view of Theorem 6.1 we see that (7.3) occurs if and only if $-2 f g=\alpha g$ for some $\alpha \in \mathbf{C}$.

In particular, if both $f$ and $g$ are constant functions, $N(f)+\Delta_{G}(g)$ becomes an equicontinuous generator and the associated Cauchy problem (7.1) is solved by power series. This case was discussed in [4] by a different method. The case of $N(f)$ and $\Delta_{G}(g)$ commuting is included in Proposition 7.1 since $\left[N(f), \Delta_{G}(g)\right]=0$ if and only if $f g=0$. A particular case is the adjoint of Kuo-Fourier-Mehler transformations of which infinitesimal generator is $i N+(i / 2) \Delta_{G}$.

We have another type of result based on the following rather obvious fact.

LEMMA 7.2. Let $\mathfrak{X}$ be an arbitrary barelled locally convex space as in §. If $\Xi \in \mathcal{L}(\mathfrak{X}, \mathfrak{X})$ is an equicontinuous generator, so is $G^{-1} \Xi G$ for any $G \in G L(\mathfrak{X})$.

Hence, if we find $G \in G L((E))$ such that $G^{-1} N(f) G=N(f)+\Delta_{G}(g)$, we can immediately conclude that $N(f)+\Delta_{G}(g)$ is an equicontinuous generator whenever so is $N(f)$. In case of $f=g=1$ such $G$ is found in [3] and $[15, \S 5.4]$.

Proposition 7.3. Let $M_{f} \in \mathcal{M}\left(E_{\mathbf{C}}, E_{\mathbf{C}}\right)$ be an equicontinuous generator and let $M_{g} \in \mathcal{M}\left(E_{\mathbf{C}}, E_{\mathbf{C}}^{*}\right)$ be arbitrary. If there exists $M \in \mathcal{L}\left(E_{\mathbf{C}}, E_{\mathbf{C}}^{*}\right)$ such that $M M_{f}=M_{g}$, then $N(f)+\Delta_{G}(g)$ is an equicontinuous generator.

Proof. In general, for any $M \in \mathcal{L}\left(E_{\mathbf{C}}, E_{\mathbf{C}}^{*}\right)$ there exists a unique operator $G \in \mathcal{L}((E),(E))$ such that

$$
G \phi_{\xi}=\exp \left(-\frac{1}{2}\langle M \xi, \xi\rangle\right) \phi_{\xi}, \quad \xi \in E_{\mathbf{C}}
$$


This is verified by a simple application of the characterization theorem of an operator symbol $[19, \S 4.4]$. Moreover, $G \in G L((E))$ since $G^{-1}$ is defined by a similar formula as in (7.4) obtained by replacing the minus sign with the plus sign. Now suppose $f$ and $g$ are as stated in the assertion. Then we have immediately

$$
G \Delta_{G}(g)=\Delta_{G}(g) G .
$$

While, by a straightforward computation based on the Wiener-Itô expansion we obtain

$$
G N(f) \phi_{\xi}=-\left\langle M M_{f} \xi, \xi\right\rangle G \phi_{\xi}+N(f) G \phi_{\xi}, \quad \xi \in E_{\mathbf{C}} .
$$

We take $M \in \mathcal{L}\left(E_{\mathbf{C}}, E_{\mathbf{C}}^{*}\right)$ as described in the statement. Then (7.6) becomes

$$
\begin{aligned}
G N(f) \phi_{\xi} & =-\left\langle M_{g} \xi, \xi\right\rangle G \phi_{\xi}+N(f) G \phi_{\xi} \\
& =-\Delta_{G}(g) G \phi_{\xi}+N(f) G \phi_{\xi}, \quad \xi \in E_{\mathbf{C}} .
\end{aligned}
$$

Hence

$$
G N(f)=\left(-\Delta_{G}(g)+N(f)\right) G .
$$

In view of (7.5) and (7.7) we obtain $G \Delta_{G}(g)+G N(f)=N(f) G$, that is

$$
\Delta_{G}(g)+N(f)=G^{-1} N(f) G .
$$

Since $N(f)$ is an equicontinuous generator by Theorem 5.5, it follows from Lemma 7.2 that $N(f)+\Delta_{G}(g)$ is also an equicontinuous generator.

It is not known whether or not $N(f)+\Delta_{G}(g)$ is an equicontinuous generator for an arbitrary $f$ with $M_{f} \in \mathcal{M}\left(E_{\mathbf{C}}, E_{\mathbf{C}}\right)$ being an equicontinuous generator and for arbitrary $g$ with $M_{g} \in \mathcal{M}\left(E_{\mathbf{C}}, E_{\mathbf{C}}^{*}\right)$.

On the other hand, the case of $f=g$ is interesting from another aspect. In view of $x(t)=a_{t}+a_{t}^{*}$ we obtain

$$
N(f)+\Delta_{G}(f)=\int_{\mathbf{R}} f(t)\left(a_{t}^{*}+a_{t}\right) a_{t} d t=\int_{\mathbf{R}} f(t) x(t) a_{t} d t .
$$

This is a first order differential operator with distribution coefficients discussed in $[19, \S 5.1]$. Hence the corresponding one-parameter subgroup is related to a one-parameter transformation group on the infinite dimensional space $E^{*}$. For example, for $f(t) \equiv 1$ we know that $N+\Delta_{G}$ is the infinitesimal generator of a homogeneous dilation: $x \mapsto e^{\theta} x$ for $x \in E^{*}$ and $\theta \in \mathbf{R}$, and the lift to $(E)$ admits a holomorphic extention (with respect to $\theta)$. Further study in this direction will appear elsewhere. 


\section{REFERENCES}

[1] L. Accardi, Yang-Mills equations and Lévy Laplacians, in "Dirichlet Forms and Stochastic Processes (Z. M. Ma et al. eds.)," pp. 1-24, Walter de Gruyter, Berlin, 1995.

[2] A. Arai and I. Mitoma, De Rham-Hodge-Kodaira decomposition in $\infty$-dimensions, Math. Ann., 291 (1991), 51-73.

[3] D. M. Chung and U. C. Ji, Cauchy problems for a partial differential equation in white noise analysis, J. Korean Math. Soc., 33 (1996), 309-318.

[4] D. M. Chung and U. C. Ji, Transformation groups on white noise functionals and their applications, to appear in Appl. Math. Optim.

[5] D. M. Chung and U. C. Ji, Some Cauchy problems in white noise analysis and associated semigroups of operators, to appear in Stoch. Anal. Appl.

[6] D. M. Chung and U. C. Ji, Transforms on white noise functionals with their applications to Cauchy problems, Nagoya Math. J., 147 (1997), 1-23.

[7] I. Dôku, On the Laplacian on a space of white noise functionals, Tsukuba J. Math., 19 (1995), 93-119.

[8] I. Dôku, H.-H. Kuo and Y.-J. Lee, Fourier transform and heat equation in white noise analysis, in "Stochastic Analysis on Infinite Dimensional Spaces (H. Kunita and H.-H. Kuo, eds.)," pp. 60-74, Longman, 1994.

[9] L. Gross, Potential theory on Hilbert space, J. Funct. Anal., 1 (1967), 123-181.

[10] T. Hida, "Analysis of Brownian Functionals," Carleton Math. Lect. Notes no. 13, Carleton University, Ottawa, 1975.

[11] T. Hida, H.-H. Kuo and N. Obata, Transformations on white noise functionals, J. Funct. Anal., 111 (1993), 259-277.

[12] T. Hida, N. Obata and K. Saitô, Infinite dimensional rotations and Laplacians in terms of white noise calculus, Nagoya Math. J., 128 (1992), 65-93.

[13] H.-H. Kuo, On Laplacian operators of generalized Brownian functionals, in "Stochastic Processes and Applications (K. Itô and T. Hida, eds.)," pp. 119-128, Lect. Notes in Math. Vol. 1203, Springer-Verlag, 1986.

[14] H.-H. Kuo, Fourier-Mehler transforms in white noise analysis, in "Gaussian Random Fields (K. Itô and T. Hida eds.)," pp. 257-271, World Scientific, 1991.

[15] H.-H. Kuo, Lectures on white noise analysis, Soochow J. Math., 18 (1992), 229-300.

[16] H.-H. Kuo, "White Noise Distribution Theory," CRC Press, 1996.

[17] P. Lévy, "Problèmes Concrets d'Analyse Fonctionelle," Gauthier-Villars, Paris, 1951.

[18] N. Obata, Rotation-invariant operators on white noise functionals, Math. Z., 210 (1992), 69-89.

[19] N. Obata, "White Noise Calculus and Fock Space," Lect. Notes in Math. Vol. 1577, Springer-Verlag, 1994.

[20] N. Obata, Lie algebras containing infinite dimensional Laplacians, in "Probability Measures on Groups and Related Structures (H. Heyer ed.)," pp. 260-273, World Scientific, 1995. 
[21] N. Obata, Constructing one-parameter transformations on white noise functions in terms of equicontinuous generators, Monatsh. Math., 124 (1997), 317-335.

[22] M. A. Piech, A fundamental solution of the parabolic equation on Hilbert space, J. Funct. Anal., 3 (1969), 85-114.

[23] M. A. Piech, Parabolic equations associated with the number operator, Trans. Amer. Math. Soc., 194 (1974), 213-222.

[24] Y. Umemura, On the infinite dimensional Laplacian operator, J. Math. Kyoto Univ., 4 (1965), 477-492.

[25] Y. Umemura and N. Kôno, Infinite dimensional Laplacian and spherical harmonics, Publ. RIMS, 1 (1966), 163-186.

[26] K. Yosida, "Functional Analysis (6th ed.)," Springer-Verlag, 1980.

\author{
Dong Myung Chung \\ Department of Mathematics \\ Sogang University \\ Seoul, 121-742 \\ Korea \\ dmchung@ccs.sogang. ac.kr \\ Un Cig Ji \\ Department of Mathematics \\ Sogang University \\ Seoul, 121-742 \\ Korea \\ ucji@nuri.net \\ Nobuaki Obata \\ Graduate School of Polymathematics \\ Nagoya University \\ Nagoya, 464-8602 \\ Japan \\ obata@math.nagoya-u.ac.jp
}

